Document downloaded from:

http://hdl.handle.net/10251/57052

This paper must be cited as:

Malik, SB.; Rueda, LC.; Thome, N. (2014). Further properties on the core partial order and other matrix partial orders. Linear and Multilinear Algebra. 62(12):1629-1648. doi:10.1080/03081087.2013.839676.

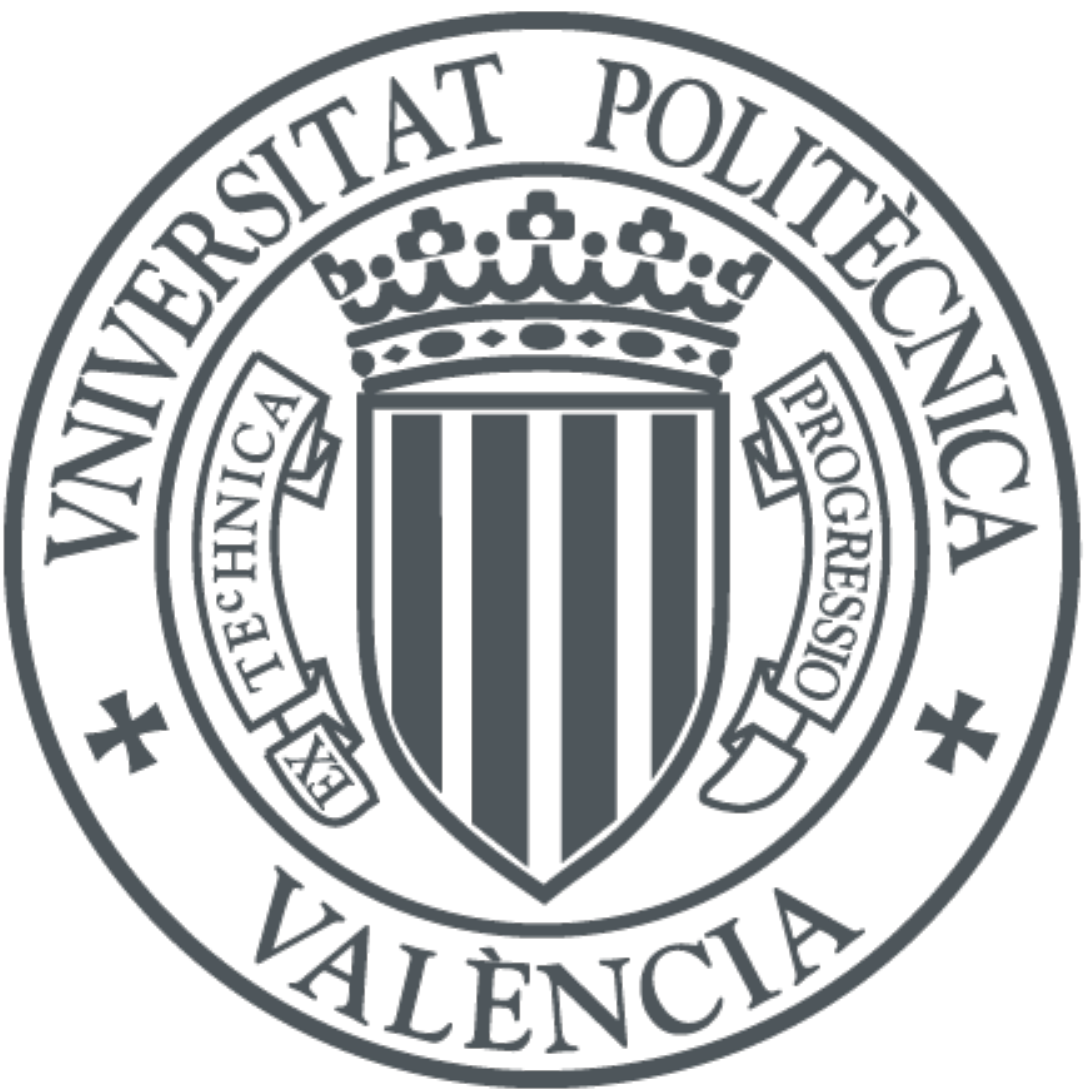

The final publication is available at

http://dx.doi.org/10.1080/03081087.2013.839676

Copyright Taylor \& Francis

Additional Information 


\title{
Properties on the core partial order and other matrix partial orders
}

\author{
S. B. Malik* $\quad$ L. Rueda ${ }^{\dagger} \quad$ N. Thome ${ }^{\ddagger}$
}

\begin{abstract}
This paper carries further the study of core partial order initiated by O.M. Baksalary and G. Trenkler in [Core inverse of matrices, Linear and Multilinear Algebra 58 (2010), 681-697]. We have extensively studied the core partial order and some new characterizations are obtained in this paper. In addition, simple expressions for the already known characterizations of the minus, the star (and one-sided star), the sharp (and onesided sharp) and the diamond partial orders are also obtained by using a Hartwig-Spindelböck decomposition.
\end{abstract}

Keywords: Minus partial order; core partial order; core inverse; matrix decomposition.

AMS subject classification: Primary: 15A09; Secondary: 06A06

\section{Introduction and Preliminaries}

The symbol $\mathbb{C}^{m \times n}$ stands for the set of $m \times n$ complex matrices. The symbols $A^{*}, \mathcal{R}(A)$, and $r k(A)$ will denote the conjugate transpose, the range and the rank of a matrix $A \in \mathbb{C}^{m \times n}$, respectively. Moreover, $I_{n}$ will denote the identity matrix of order $n \times n$.

A square matrix $A$ is said to have index at most 1 if $r k\left(A^{2}\right)=r k(A)$. This condition characterizes the existence of a matrix $X$ that satisfies $A X A=A$, $X A X=X$, and $A X=X A$. This (unique) matrix is called the group inverse of $A$ and denoted by $X=A^{\#}$. If $A \in \mathbb{C}^{m \times n}$, a matrix $X \in \mathbb{C}^{n \times m}$ that only satisfies the equation $A X A=A$ is called a $\{1\}$-inverse of $A$. If $X$ satisfies $A X A=A, X A X=X$ and moreover $A X$ and $X A$ are hermitian, $X$ is called the Moore-Penrose of $A$. The Moore-Penrose of $A$ always exists, it is unique

\footnotetext{
*School of Liberal Studies, Ambedkar University, Kashmere Gate, Delhi, India. E-mail: saroj.malik@gmail.com.

${ }^{\dagger}$ Departamento e Instituto de Matemática. Universidad Nacional del Sur, 8000, Bahía Blanca, Argentina. E-mail: laura.rueda@uns.edu.ar.

¥Instituto Universitario de Matemática Multidisciplinar, Universitat Politècnica de València, 46022, València, Spain. E-mail: njthome@mat.upv.es. This author was partially supported by Ministry of Education of Spain (grant DGI MTM2010-18228) and by Universidad Nacional de La Pampa, Argentina, Facultad de Ingeniería (grant Resol. No 049/11).
} 
and will be denoted by $X=A^{\dagger}$. For further properties of these inverses we refer the reader to $[6,7]$.

It is well known that (see, for example, $[15,2,11]$ ), for $A, B \in \mathbb{C}^{n \times n}$,

- the minus partial order is defined as: $A \leq B$ if and only if there exists a $\{1\}$-inverse $A^{-}$of $A$ such that $A^{-} A=A^{-} B$ and $A A^{-}=B A^{-}$;

- the star partial order as: $A \stackrel{*}{\leq} B$ if and only if $A^{*} A=A^{*} B$ and $A A^{*}=$ $B A^{*}$;

- the sharp partial order as: if $A, B$ have index at most 1 then $A \stackrel{\#}{\leq} B$ if and only if $A^{\#} A=A^{\#} B$ and $A A^{\#}=B A^{\#}$;

- the diamond partial order as: $A \stackrel{\hat{\leq}}{\leq} B$ if and only if $\mathcal{R}(A) \subseteq \mathcal{R}(B), \mathcal{R}\left(A^{*}\right) \subseteq$ $\mathcal{R}\left(B^{*}\right)$, and $A A^{*} A=A B^{*} A$.

For each of partial orders that we work in this article (e.g., minus, star, sharp, etc.) it is easy to see that if the upper matrix is the zero matrix, the lower is also zero. That is, if $\stackrel{?}{\leq}$ represents any of these partial orders, $A \stackrel{?}{\leq} B$ with $B=O$ implies $A=O$. So, we will assume in the rest of the paper that the upper matrix is non-zero.

In [4], O.M. Baksalary and G. Trenkler introduced and studied in detail a generalized inverse called the core inverse. For $A \in \mathbb{C}^{n \times n}$, the core inverse of $A$ is the matrix $X \in \mathbb{C}^{n \times n}$ satisfying the relations $A X=A A^{\dagger}$ and $\mathcal{R}(X) \subseteq \mathcal{R}(A)$, and is denoted by $X=A^{\boxplus}$. The authors proved that the core inverse of $A$ exists if and only if $A$ has index at most 1 and, whenever it exists, is unique.

In the literature there are several papers which study properties of the matrix orders defined above and the involved generalized inverses among others. For instance, $[1,8,16,17,18,19]$ are a few references where some properties can be found.

In what follows, we will consider a Hartwig-Spindelböck decomposition of a square matrix $[10,3]$. For any matrix $B \in \mathbb{C}^{n \times n}$ of rank $r>0$ this decomposition is given by

$$
B=U\left[\begin{array}{cc}
\Sigma K & \Sigma L \\
O & O
\end{array}\right] U^{*}
$$

where $U \in \mathbb{C}^{n \times n}$ is unitary, $\Sigma \in \mathbb{C}^{r \times r}$ is a diagonal positive definite matrix and $K \in \mathbb{C}^{r \times r}, L \in \mathbb{C}^{r \times(n-r)}$ satisfy the condition $K K^{*}+L L^{*}=I_{r}$. Specifically, $\Sigma=\operatorname{diag}\left(\sigma_{1} I_{r_{1}}, \ldots, \sigma_{t} I_{r_{t}}\right)$ is a diagonal matrix, the diagonal entries $\sigma_{i}$ being singular values of $B, \sigma_{1}>\sigma_{2}>\ldots>\sigma_{t}>0, r_{1}+r_{2}+\ldots+r_{t}=r$, however this last information about $\sigma_{i}$ will be not relevant in our investigation.

By using decomposition (1), the successors of a given matrix under the core partial order were found in [4, Lemma 3] in the set of matrices possessing core inverse. Our main aim is to exploit this decomposition to analyze predecessors for a fixed element under the core partial order in the set of matrices that have core inverse. Furthermore, a similar technique is used to study the other aforementioned partial orders and the one-sided ones. 
This paper is organized as follows. In Section 2, we first characterize matrices below a given matrix under the four major partial orders namely the minus, the star, the sharp and the diamond partial orders, using the Hartwig-Spindelböck decomposition of the upper matrix. Section 3 deals with studying one-sided star and one-sided sharp partial orders. Here we give simple expressions for their already known characterizations. In Section 4, we study in detail the core partial order. Some characterizations are developed as well as the case of $E P$ matrices and the reverse order law. Finally, it is well known for the minus, the star, and the sharp orders that $A$ is below $B$ if and only if $B-A$ is below $B$. In general this property is not possessed by core partial order. In Section 5 , we give some conditions under which each one of them implies the other one.

\section{Minus, star, sharp and diamond partial orders}

We recall that it is well known [9] that the minus partial order is characterized as follows: if $A, B \in \mathbb{C}^{n \times n}$ then

$$
A \stackrel{-}{\leq} B \quad \Longleftrightarrow \quad r k(B-A)=r k(B)-r k(A)
$$

Next result characterizes predecessors of a given matrix under the minus partial order, that is, for a given matrix $B$ we find matrices $A$ such that $A \leq B$.

Theorem 1 Let $B \in \mathbb{C}^{n \times n}$ be a non-zero matrix written as in (1). The following conditions are equivalent.

(a) There exists a matrix $A \in \mathbb{C}^{n \times n}$ such that $A \stackrel{-}{\leq} B$.

(b) There exists an idempotent matrix $T \in \mathbb{C}^{r \times r}$ such that

$$
A=U\left[\begin{array}{cc}
T \Sigma K & T \Sigma L \\
O & O
\end{array}\right] U^{*} .
$$

Proof. (a) $\Longrightarrow$ (b) Let $A \in \mathbb{C}^{n \times n}$ be such that $A \leq B$. It is easy to check that expression (1) of $B$ can be re-written as

$$
B=U\left[\begin{array}{ll}
I_{r} & O \\
O & O
\end{array}\right]\left[\begin{array}{cc}
\Sigma K & \Sigma L \\
O & O
\end{array}\right] U^{*}
$$

We denote

$$
P=U\left[\begin{array}{ll}
I_{r} & O \\
O & O
\end{array}\right], \quad Q=\left[\begin{array}{cc}
\Sigma K & \Sigma L \\
O & O
\end{array}\right] U^{*}
$$

and partition

$$
P=\left[\begin{array}{ll}
P_{1} & O
\end{array}\right], \quad Q=\left[\begin{array}{c}
Q_{1} \\
O
\end{array}\right]
$$


with $P_{1} \in \mathbb{C}^{n \times r}$ and $Q_{1} \in \mathbb{C}^{r \times n}$. So, matrix $B$ can be expressed as

$$
B=\left[\begin{array}{ll}
P_{1} & O
\end{array}\right]\left[\begin{array}{c}
Q_{1} \\
O
\end{array}\right]=P_{1} Q_{1}
$$

where $r k\left(P_{1}\right)=r=r k(B)$, and since $K K^{*}+L L^{*}=I_{r}$,

$$
r k\left(Q_{1}\right)=r k(Q)=r k\left(\left[\begin{array}{ll}
\Sigma K & \Sigma L
\end{array}\right]\right)=r k\left(\left[\begin{array}{ll}
K & L
\end{array}\right]\left[\begin{array}{ll}
K & L
\end{array}\right]^{*}\right)=r .
$$

Thus, $B=P_{1} Q_{1}$ is a full rank factorization of the matrix $B$. Since $A \leq B$ and $B \neq O$, Theorem 3.4.6 in [15] asserts that there exists an idempotent matrix $T \in \mathbb{C}^{r \times r}$ such that $A=P_{1} T Q_{1}$. Using (4), simple computations lead to

$$
A=\left[\begin{array}{ll}
P_{1} & O
\end{array}\right]\left[\begin{array}{ll}
T & O \\
O & O
\end{array}\right]\left[\begin{array}{c}
Q_{1} \\
O
\end{array}\right]=U\left[\begin{array}{cc}
T \Sigma K & T \Sigma L \\
O & O
\end{array}\right] U^{*}
$$

(b) $\Longrightarrow$ (a) From (1) and (3) we obtain

$$
U^{*}(B-A) U=\left[\begin{array}{cc}
\left(I_{r}-T\right) \Sigma K & \left(I_{r}-T\right) \Sigma L \\
O & O
\end{array}\right] .
$$

Since $K K^{*}+L L^{*}=I_{r}$, we have that

$$
r k\left(\Sigma\left[\begin{array}{ll}
K & L
\end{array}\right]\right)=r k\left(\left[\begin{array}{ll}
K & L
\end{array}\right]\left[\begin{array}{ll}
K & L
\end{array}\right]^{*}\right)=r
$$

from where it is easy to see that

$$
U^{*}(B-A) U=\left[\begin{array}{cc}
I_{r}-T & O \\
O & O
\end{array}\right]\left[\begin{array}{cc}
\Sigma K & \Sigma L \\
R & S
\end{array}\right]
$$

for some $R \in \mathbb{C}^{(n-r) \times r}$ and $S \in \mathbb{C}^{(n-r) \times(n-r)}$ such that the matrix $\left[\begin{array}{cc}\Sigma K & \Sigma L \\ R & S\end{array}\right]$ is nonsingular. Thus, $r k(B-A)=r k\left(I_{r}-T\right)=r-r k(T)$ because of $T$ is idempotent.

On the other hand, one has

$$
r k(A)=r k\left(U\left[\begin{array}{ll}
T & O \\
O & O
\end{array}\right]\left[\begin{array}{cc}
\Sigma K & \Sigma L \\
R & S
\end{array}\right] U^{*}\right)=r k(T) .
$$

Then, $r k(B)-r k(A)=r-r k(T)$. Hence, equivalence (2) guarantees that $A \leq B$

Remark 2 For every matrix $A \in \mathbb{C}^{n \times n}$ such that $A \leq B$ in (a) of Theorem 1, there exists only one matrix $T \in \mathbb{C}^{r \times r}$ in the conditions indicated in (b). Indeed, in order to show the uniqueness of $T$, suppose that $T_{1}$ is also an idempotent matrix such that

$$
A=U\left[\begin{array}{cc}
T_{1} \Sigma K & T_{1} \Sigma L \\
O & O
\end{array}\right] U^{*} .
$$

So, $T \Sigma K=T_{1} \Sigma K$ and $T \Sigma L=T_{1} \Sigma L$. Post-multiplying these last equalities by $K^{*}$ and $L^{*}$, respectively, and then adding we get $T=T_{1}$ because $\Sigma$ is nonsingular. 
The form for the matrix $A$ obtained in Theorem 1 will play an important role in the whole paper and so, we will label it as follows. Let $A \in \mathbb{C}^{n \times n}$ be a matrix given by

$$
A=U\left[\begin{array}{cc}
T \Sigma K & T \Sigma L \\
O & O
\end{array}\right] U^{*}
$$

where $U \in \mathbb{C}^{n \times n}$ is unitary, $\Sigma \in \mathbb{C}^{r \times r}$ is diagonal positive definite, $T \in \mathbb{C}^{r \times r}$, and moreover $K \in \mathbb{C}^{r \times r}$ and $L \in \mathbb{C}^{r \times(n-r)}$ satisfy $K K^{*}+L L^{*}=I_{r}$.

Next result provides the Moore-Penrose and group inverse of a matrix $A$ as in $(5)$.

Lemma 3 Let $A \in \mathbb{C}^{n \times n}$ be a matrix as in (5). Then

(a) the Moore-Penrose inverse of $A$ is given by

$$
A^{\dagger}=U\left[\begin{array}{cc}
K^{*}(T \Sigma)^{\dagger} & O \\
L^{*}(T \Sigma)^{\dagger} & O
\end{array}\right] U^{*}
$$

(b) if $K$ is nonsingular and $T \Sigma K$ has index at most 1 then there exists the group inverse of $A$ and is given by

$$
A^{\#}=U\left[\begin{array}{cc}
(T \Sigma K)^{\#} & (T \Sigma K)^{\#} K^{-1} L \\
O & O
\end{array}\right] U^{*}
$$

In particular, if $T^{2}=T$ and $T \Sigma K=\Sigma K T$ then $T \Sigma K$ has index at most 1 and $(T \Sigma K)^{\#}=(\Sigma K)^{-1} T$.

Proof. (a) It is easy to check that $A A^{\dagger} A=A, A^{\dagger} A A^{\dagger}=A^{\dagger},\left(A A^{\dagger}\right)^{*}=A A^{\dagger}$, and $\left(A^{\dagger} A\right)^{*}=A^{\dagger} A$ hold.

(b) Since $\mathcal{R}(T \Sigma L) \subseteq \mathcal{R}(T)=\mathcal{R}\left(T \Sigma K(T \Sigma K)^{\#}\right)$, we have that the range of $T \Sigma L$ is included into the null space of $I_{r}-T \Sigma K(T \Sigma K)^{\#}$, that is $\left(I_{r}-\right.$ $\left.T \Sigma K(T \Sigma K)^{\#}\right) T \Sigma L=O$.

Now, it follows from [7, Theorem 7.7.3, pp. 143] that the group inverse of $A$ is given by

$$
U^{*} A^{\#} U=\left[\begin{array}{cc}
(T \Sigma K)^{\#} & \left((T \Sigma K)^{\#}\right)^{2} T \Sigma L \\
O & O
\end{array}\right]=\left[\begin{array}{cc}
(T \Sigma K)^{\#} & (T \Sigma K)^{\#} K^{-1} L \\
O & O
\end{array}\right]
$$

because $\left((T \Sigma K)^{\#}\right)^{2} T \Sigma K=(T \Sigma K)^{\#} T \Sigma K(T \Sigma K)^{\#}=(T \Sigma K)^{\#}$. The proof of $(T \Sigma K)^{\#}=(\Sigma K)^{-1} T$ in the mentioned particular case follows directly by definition.

We recall that the star partial order is characterized as follows: if $A, B \in$ $\mathbb{C}^{n \times n}$ then

$$
A \stackrel{*}{\leq} B \quad \Longleftrightarrow \quad A^{\dagger} A=A^{\dagger} B \text { and } A A^{\dagger}=B A^{\dagger} .
$$

Now, we characterize predecessors under the star partial order.

Theorem 4 Let $B \in \mathbb{C}^{n \times n}$ be a non-zero matrix written as in (1). The following conditions are equivalent. 
(a) There exists a matrix $A \in \mathbb{C}^{n \times n}$ such that $A \stackrel{*}{\leq} B$.

(b) There exists an idempotent matrix $T \in \mathbb{C}^{r \times r}$ such that $A$ has the form (5) and, moreover, one of the following equivalent conditions holds:

(i) $T \Sigma^{2}=\Sigma^{2} T$ and $T$ is hermitian.

(ii) $(T \Sigma)^{\dagger}=\Sigma^{-1} T$.

(iii) $\left(\left(I_{r}-T\right) \Sigma\right)^{\dagger}=\Sigma^{-1}\left(I_{r}-T\right)$.

Proof. We first prove the equivalence of (a) and (b) when (ii) in (b) holds.

(a) $\Longrightarrow$ (b) (ii) Suppose that $A \stackrel{*}{\leq} B$. By Theorem 5.2.10 in [15] $A \stackrel{-}{\leq} B$ and $B A^{\dagger} B=A$. By Theorem 1 , there exists an idempotent matrix $T \in \mathbb{C}^{r \times r}$ such that

$$
A=U\left[\begin{array}{cc}
T \Sigma K & T \Sigma L \\
O & O
\end{array}\right] U^{*}
$$

By Lemma 3,

$$
A^{\dagger}=U\left[\begin{array}{cc}
K^{*}(T \Sigma)^{\dagger} & O \\
L^{*}(T \Sigma)^{\dagger} & O
\end{array}\right] U^{*}
$$

Substituting these expressions in $B A^{\dagger} B=A$ we have

$$
\begin{aligned}
\Sigma(T \Sigma)^{\dagger} \Sigma K & =T \Sigma K \\
\Sigma(T \Sigma)^{\dagger} \Sigma L & =T \Sigma L .
\end{aligned}
$$

Post-multiplying (8) and (9) by $K^{*}$ and $L^{*}$, respectively, and then adding we have $\Sigma(T \Sigma)^{\dagger}=T$ and therefore, $(T \Sigma)^{\dagger}=\Sigma^{-1} T$.

(b) (ii) $\Longrightarrow$ (a) Using Lemma 3 (a), it is easy to verify that $A^{\dagger} A=A^{\dagger} B$ and $A A^{\dagger}=B A^{\dagger}$.

(b) (i) $\Longrightarrow$ (b) (ii) It follows from the definition of Moore-Penrose inverse.

(b) (ii) $\Longrightarrow$ (b) (i) Pre-multiplying $(T \Sigma)^{\dagger}=\Sigma^{-1} T$ by $T \Sigma$ and using that $T$ is idempotent we get $T \Sigma(T \Sigma)^{\dagger}=T$, so $T$ is hermitian.

On the other hand, post-multiplying $(T \Sigma)^{\dagger}=\Sigma^{-1} T$ by $T \Sigma$ we get $(T \Sigma)^{\dagger} T \Sigma=$ $\Sigma^{-1} T \Sigma$ from where $\Sigma T^{*} \Sigma^{-1}=\Sigma^{-1} T \Sigma$ and thus $T \Sigma^{2}=\Sigma^{2} T$.

The proof of the equivalence of (a) and (b) when (iii) in (b) holds follows on similar lines in view of the following equivalence: $A \stackrel{*}{\leq} B$ if and only if $B-A \stackrel{*}{\leq} B$ (see [15, Theorem 5.2.3]).

We close this section with a result analogous to Theorem 1 and Theorem 4 related to the sharp partial order and other characterizing the diamond partial order.

Theorem 5 Let $B \in \mathbb{C}^{n \times n}$ be a non-zero matrix written as in (1) with $K$ nonsingular. The following conditions are equivalent.

(a) There exists a matrix $A \in \mathbb{C}^{n \times n}$ of index at most 1 such that $A \stackrel{\#}{\leq} B$.

(b) There exists an idempotent matrix $T \in \mathbb{C}^{r \times r}$ that commutes with $\Sigma K$ and $A$ has the form (5). 
Proof. By [15, Corollary 6.3.14], $A \stackrel{\#}{\leq} B$ if and only if $A \overline{\leq} B$ and $A B=B A$ provided that $A$ and $B$ have index at most 1. Now, a direct application of Theorem 1 gives the equivalence of $A \overline{\leq} B$ and the expression (5) for $A$ and simple computations show the equivalence of $A B=B A$ and $T \Sigma K=\Sigma K T$. Notice that in (b) $\Longrightarrow$ (a) the existence of $A^{\#}$ is guaranteed by Lemma 3 (b).

Theorem 6 Let $B \in \mathbb{C}^{n \times n}$ be a non-zero matrix written as in (1). The following conditions are equivalent.

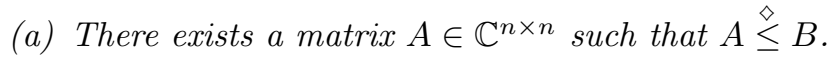

(b) There exists an idempotent matrix $T \in \mathbb{C}^{r \times r}$ such that

$$
A=U\left[\begin{array}{cc}
\left(\Sigma^{-1} T\right)^{\dagger} K & \left(\Sigma^{-1} T\right)^{\dagger} L \\
O & O
\end{array}\right] U^{*} .
$$

Proof. By Theorem 2 in [2], $A \stackrel{\vec{x}}{\leq}$ if and only if $A^{\dagger} \overline{\leq} B^{\dagger}$, which is equivalent to $\left(A^{\dagger}\right)^{*} \leq\left(B^{\dagger}\right)^{*}$ by $(2)$. By Theorem 1 , there exists an idempotent matrix $T^{*} \in$ $\mathbb{C}^{r \times r}$ such that $\left(A^{\dagger}\right)^{*}=U\left[\begin{array}{cc}T^{*} \Sigma^{-1} K & T^{*} \Sigma^{-1} L \\ O & O\end{array}\right] U^{*}$. Taking into account the equalities $\left(A^{\dagger}\right)^{*}=\left(A^{*}\right)^{\dagger}$ and $\left(\left(A^{*}\right)^{\dagger}\right)^{\dagger}=A^{*}$, by Lemma 3 , we have that

$$
A^{*}=U\left[\begin{array}{cc}
K^{*}\left(T^{*} \Sigma^{-1}\right)^{\dagger} & O \\
L^{*}\left(T^{*} \Sigma^{-1}\right)^{\dagger} & O
\end{array}\right] U^{*}
$$

which gives the form (10).

\section{One-sided star and one-sided sharp partial or- ders}

We recall that right and left star partial orders are respectively defined in [15] as follows: if $A, B \in \mathbb{C}^{n \times n}$ then

$$
A \leq * B \quad \Longleftrightarrow \quad A A^{*}=B A^{*} \text { and } \mathcal{R}\left(A^{*}\right) \subseteq \mathcal{R}\left(B^{*}\right)
$$

and

$$
A * \leq B \quad \Longleftrightarrow \quad A^{*} A=A^{*} B \text { and } \mathcal{R}(A) \subseteq \mathcal{R}(B) .
$$

Theorem 7 Let $B \in \mathbb{C}^{n \times n}$ be a non-zero matrix written as in (1). The following conditions are equivalent.

(a) There exists a matrix $A \in \mathbb{C}^{n \times n}$ such that $A \leq * B$.

(b) There exists an idempotent matrix $T \in \mathbb{C}^{r \times r}$ such that $A$ has the form (5) and $T \Sigma^{2}=\Sigma^{2} T^{*}$. 
Proof. By Theorem 6.5.25 in [15], $A \leq * B$ if and only if $A \leq B$ and $B^{\dagger} A$ is hermitian. By Theorem 1, there exists an idempotent matrix $T \in \mathbb{C}^{r \times r}$ such that $A$ has the form (5) required in item (b) and, by [4, expression (1.13)],

$$
B^{\dagger}=U\left[\begin{array}{cc}
K^{*} \Sigma^{-1} & O \\
L^{*} \Sigma^{-1} & O
\end{array}\right] U^{*}
$$

Since $B^{\dagger} A$ is hermitian if and only if

$$
\left[\begin{array}{cc}
K^{*} \Sigma^{-1} T \Sigma K & K^{*} \Sigma^{-1} T \Sigma L \\
L^{*} \Sigma^{-1} T \Sigma K & L^{*} \Sigma^{-1} T \Sigma L
\end{array}\right]=\left[\begin{array}{cc}
K^{*} \Sigma T^{*} \Sigma^{-1} K & K^{*} \Sigma T^{*} \Sigma^{-1} L \\
L^{*} \Sigma T^{*} \Sigma^{-1} K & L^{*} \Sigma T^{*} \Sigma^{-1} L
\end{array}\right]
$$

we obtain the following system

$$
\begin{aligned}
K^{*} \Sigma^{-1} T \Sigma K & =K^{*} \Sigma T^{*} \Sigma^{-1} K, \\
K^{*} \Sigma^{-1} T \Sigma L & =K^{*} \Sigma T^{*} \Sigma^{-1} L, \\
L^{*} \Sigma^{-1} T \Sigma K & =L^{*} \Sigma T^{*} \Sigma^{-1} K, \\
L^{*} \Sigma^{-1} T \Sigma L & =L^{*} \Sigma T^{*} \Sigma^{-1} L .
\end{aligned}
$$

Post-multiplying (11) and (12) by $K^{*}$ and $L^{*}$, respectively, and then adding we have

$$
K^{*} \Sigma^{-1} T \Sigma=K^{*} \Sigma T^{*} \Sigma^{-1} .
$$

Similarly, from (13) and (14) we obtain

$$
L^{*} \Sigma^{-1} T \Sigma=L^{*} \Sigma T^{*} \Sigma^{-1} .
$$

Pre-multiplying (15) and (16) by $K$ and $L$ respectively, and then adding we have $\Sigma^{-1} T \Sigma=\Sigma T^{*} \Sigma^{-1}$ or equivalently $T \Sigma^{2}=\Sigma^{2} T^{*}$.

Similarly,

Theorem 8 Let $B \in \mathbb{C}^{n \times n}$ be a non-zero matrix written as in (1). The following conditions are equivalent.

(a) There exists a matrix $A \in \mathbb{C}^{n \times n}$ such that $A * \leq B$.

(b) There exists an idempotent hermitian matrix $T \in \mathbb{C}^{r \times r}$ such that $A$ has the form (5).

Proof. By Remark 6.5.13 in [15], $A * \leq B$ if and only if $A^{*} \leq * B^{*}$, which is equivalent to $A^{*} \leq B^{*}$ and $\left(B^{*}\right)^{\dagger} A^{*}$ is hermitian. Since $A^{*} \leq B^{*}$ is equivalent to $A \overline{\leq} \leq B$, and $\left(B^{*}\right)^{\dagger}=\left(B^{\dagger}\right)^{*}$, we have $A * \leq B$ if and only if $A \overline{\leq} B$ and $A B^{\dagger}$ is hermitian. By Theorem $1, A \leq B$ if and only if there exists an idempotent matrix $T \in \mathbb{C}^{r \times r}$ such that $A$ has the form required in item (b), and we can easily obtain that $A B^{\dagger}$ is hermitian if and only if $T$ is hermitian. 
Now, we recall that it is well known [15] that right and left sharp partial orders are respectively defined as follows: if $A, B \in \mathbb{C}^{n \times n}$ are matrices of index at most 1 then

$$
A \leq \# B \quad \Longleftrightarrow \quad A A^{\#}=B A^{\#} \text { and } \mathcal{R}\left(A^{*}\right) \subseteq \mathcal{R}\left(B^{*}\right)
$$

and

$$
A \# \leq B \quad \Longleftrightarrow \quad A^{\#} A=A^{\#} B \text { and } \mathcal{R}(A) \subseteq \mathcal{R}(B) .
$$

Theorem 9 Let $B \in \mathbb{C}^{n \times n}$ be a non-zero matrix written as in (1) with $K a$ nonsingular matrix. The following conditions are equivalent.

(a) There exists a matrix $A \in \mathbb{C}^{n \times n}$ of index at most 1 and $A \leq \# B$.

(b) There exists an idempotent matrix $T \in \mathbb{C}^{r \times r}$ such that $A$ has the form (5) and $T \Sigma K T=\Sigma K T$.

Proof. By Theorem 6.3.13 in [15] and taking into account that $A A^{\#}=B A^{\#}$ is equivalent to $A^{2}=B A$ we can show that $A \leq \# B$ if and only if $A \leq B$ and $A^{2}=B A$. Now, Theorem 1 shows that $A \overline{\leq} B$ is equivalent to the existence of an idempotent matrix $T \in \mathbb{C}^{r \times r}$ such that $A$ has the form given in (5). Since $\Sigma K$ is nonsingular, easy computations show that condition $A^{2}=B A$ is equivalent to $T \Sigma K T=\Sigma K T$. Moreover, by Lemma 3, this last condition assures the existence of the group inverse of $A$.

Remark 10 A simple fact of matrix analysis assures that if $X, Y$ are conformable for multiplication matrices such that $X$ is idempotent, $Y$ is nonsingular, and $X Y X=Y X$ then $X Y^{-1} X=Y^{-1} X$. Under the conditions of Theorem 9, this fact assures that $T(\Sigma K)^{-1} T=(\Sigma K)^{-1} T$. So, if $A \leq \# B$ then the definition of the group inverse allows us to check that $(T \Sigma K)^{\#}=(\Sigma K)^{-2} T \Sigma K$ and, in this case, we can write

$$
A^{\#}=U\left[\begin{array}{cc}
(\Sigma K)^{-2} T \Sigma K & (\Sigma K)^{-2} T \Sigma L \\
O & O
\end{array}\right] U^{*} .
$$

We can prove on similar lines the following:

Theorem 11 Let $B \in \mathbb{C}^{n \times n}$ be a non-zero matrix written as in (1) with $K a$ nonsingular matrix. The following conditions are equivalent.

(a) There exists a matrix $A \in \mathbb{C}^{n \times n}$ of index at most 1 such that $A \# \leq B$.

(b) There exists an idempotent matrix $T \in \mathbb{C}^{r \times r}$ such that $A$ has the form (5) and $T \Sigma K T=T \Sigma K$.

Similarly to Remark 10, in this case we get

$$
A^{\#}=U\left[\begin{array}{cc}
T(\Sigma K)^{-1} & T(\Sigma K)^{-1} K^{-1} L \\
O & O
\end{array}\right] U^{*}
$$




\section{The core partial order}

Throughout this section, all the matrices will have index at most 1 , that is we will consider matrices $B \in \mathbb{C}^{n \times n}$ such that $r k\left(B^{2}\right)=r k(B)$. Thus, if $B$ has the Hartwig-Spindelböck decomposition (1) then $K$ is a nonsingular matrix as shown in [4] and the core inverse of $B$ exists.

Lemma 12 Let $A$ be a matrix as in (5) of index at most 1 with $K$ a nonsingular matrix. Then $A$ has core inverse given by

$$
A^{\boxplus}=U\left[\begin{array}{cc}
(T \Sigma K)^{\#} T \Sigma(T \Sigma)^{\dagger} & O \\
O & O
\end{array}\right] U^{*} .
$$

Proof. By Theorem 1 (i) in [4] we have that $A^{\boxplus}=A^{\#} A A^{\dagger}$. Notice that the assumption that $A$ has index at most 1 means that $T \Sigma K$ has group inverse.

Now, Lemma 3 yields

$$
A^{\boxplus}=A^{\#} A A^{\dagger}=U\left[\begin{array}{cc}
(T \Sigma K)^{\#} T \Sigma(T \Sigma)^{\dagger} & O \\
O & O
\end{array}\right] U^{*}
$$

as desired.

Remark 13 Notice that the condition that $A$ has index at most 1 in Lemma 12, (or, in other words, $r k(T \Sigma K T)=r k(T))$ is trivially equivalent to $\mathcal{R}(T \Sigma K T)=$ $\mathcal{R}(T)$. Moreover, a sufficient condition to obtain the equality $r k(T \Sigma K T)=$ $r k(T)$ is $T=\Sigma K(T \Sigma K)^{\#} T$. In fact, pre-multiplying $T=\Sigma K(T \Sigma K)^{\#} T$ by $\Sigma K T$ and post-multiplying by $\Sigma K$ we have $\Sigma K T \Sigma K=\left(\Sigma K(T \Sigma K)^{\#} T\right) \Sigma K T \Sigma K$. Then, $\Sigma K T=T \Sigma K T$, which implies that $r k(T \Sigma K T)=r k(T)$ holds.

We need the following properties concerning the Moore-Penrose inverse.

Lemma 14 Let $X \in \mathbb{C}^{m \times m}$. Then the following statements hold:

(a) $X$ is an idempotent hermitian matrix if and only if $X=X Y(X Y)^{\dagger}$ for every nonsingular matrix $Y \in \mathbb{C}^{m \times m}$.

(b) $X$ is an idempotent hermitian matrix if and only if $(X Y)^{\dagger}=(X Y)^{\dagger} X$ for every nonsingular matrix $Y \in \mathbb{C}^{m \times m}$.

Proof.

(a) Assume that $X$ is an idempotent hermitian matrix. It is known that $P_{X}=X X^{\dagger}$ is an orthogonal projector onto $\mathcal{R}(X)$. If $Y \in \mathbb{C}^{m \times m}$ is nonsingular then $\mathcal{R}(X)=\mathcal{R}(X Y)=\mathcal{R}\left(X Y(X Y)^{\dagger}\right)$; thus $X Y(X Y)^{\dagger}$ is an orthogonal projector onto $\mathcal{R}(X)$. Then, we have $P_{X}=X Y(X Y)^{\dagger}$. Using that $X=X^{2}=X^{*}$, we have $X^{\dagger}=X$, so $P_{X}=X X^{\dagger}=X X=X$. Hence, $X=X Y(X Y)^{\dagger}$. The converse is trivial.

(b) Assume that $(X Y)^{\dagger}=(X Y)^{\dagger} X$. If we pre-multiply this equality by $X Y$, we get $X Y(X Y)^{\dagger} X=X Y(X Y)^{\dagger}$, so $X^{*} X Y(X Y)^{\dagger}=X Y(X Y)^{\dagger}$. Now, postmultiplying by $X Y$ and using that $Y$ is nonsingular we obtain $X^{*} X=X$, hence 
$X$ is an idempotent hermitian matrix. The converse follows pre-multiplying by $(X Y)^{\dagger}$ the condition $X=X Y(X Y)^{\dagger}$ in (a).

We recall that the core partial order is defined as follows [4]: if $A, B \in \mathbb{C}^{n \times n}$ have index at most 1 then

$$
A \stackrel{\oplus}{\leq} B \quad \Longleftrightarrow \quad A^{\boxplus} A=A^{\boxplus} B \text { and } A A^{\boxplus}=B A^{\boxplus}
$$

where $A^{\boxplus}=A^{\#} A A^{\dagger}$ is the core inverse of $A$.

As a first remark we present some direct characterizations of the core partial order in terms of the whole matrices. Let $A, B \in \mathbb{C}^{n \times n}$ be two matrices that have core inverse. Then the following conditions are equivalent:

(a) $A \stackrel{\oplus}{\leq} B$.

(b) $A A^{\dagger} B=B A^{\#} A=A$.

(c) $A^{\dagger} A=A^{\dagger} B$ and $A A^{\#}=B A^{\#}$.

(d) $A^{*} A=A^{*} B$ and $A A^{\#}=B A^{\#}$.

All the above equivalences can be easily obtained.

Comparing this last remark to Theorem 2.4 in [12] we observe that actually conditions on the column spaces in the one-side partial orders $\leq \#$ and $* \leq$ in that theorem can be dropped out. Item (b) above was included in Lemma 2 in [14] and that lemma also contains an equivalent form of (d).

On the other hand, next result shows that core partial order implies diamond partial order.

Theorem 15 Let $A, B \in \mathbb{C}^{n \times n}$ such that $A$ has core inverse. If $A \stackrel{\oplus}{\leq} B$ then $A \stackrel{\otimes}{\leq} B$

Proof. Since $A \stackrel{\oplus}{\leq} B$, the equalities $A^{\dagger} A=A^{\dagger} B, A A^{\#}=B A^{\#}$, and $A^{*} A=$ $A^{*} B$ hold. Then, $A=A A^{\#} A=B A^{\#} A$ implies $\mathcal{R}(A) \subseteq \mathcal{R}(B)$. From $A^{\dagger} A=$ $A^{\dagger} B=B^{*}\left(A^{\dagger}\right)^{*}$ we get $A^{\dagger}=A^{\dagger} A A^{\dagger}=B^{*}\left(A^{\dagger}\right)^{*} A^{\dagger}$. Thus, $\mathcal{R}\left(A^{*}\right)=\mathcal{R}\left(A^{\dagger}\right) \subseteq$ $\mathcal{R}\left(B^{*}\right)$. Finally, from $A^{*} A=A^{*} B$ it is clear that $A A^{*} A=A B^{*} A$. Hence, $A \stackrel{\otimes}{\leq} B$

Note that the converse is not true as the following matrices shown:

$$
A=\left[\begin{array}{ll}
1 & 0 \\
0 & 0
\end{array}\right] \quad \text { and } \quad B=\left[\begin{array}{ll}
1 & 1 \\
1 & 0
\end{array}\right]
$$

In this example, $A \stackrel{\otimes}{\leq} B$ but $A \stackrel{\oplus}{\leq} B$ does not hold.

We now characterize predecessors of a fixed matrix under the core partial order.

Theorem 16 Let $B \in \mathbb{C}^{n \times n}$ be a non-zero matrix written as in (1) such that $B$ has core inverse. Then the following statements are equivalent. 
(a) There exists a matrix $A \in \mathbb{C}^{n \times n}$ that has a core inverse such that $A \stackrel{\oplus}{\leq} B$.

(b) There exists a matrix $T \in \mathbb{C}^{r \times r}$ such that $A$ has the form (5) and, moreover, one of the following equivalent conditions holds:

(i) $T$ is an idempotent hermitian matrix and $T=\Sigma K(T \Sigma K)^{\#} T$.

(ii) $T \Sigma K T=\Sigma K T$ and $(T \Sigma)^{\dagger} T=(T \Sigma)^{\dagger}$.

(iii) $T$ is an idempotent hermitian matrix and $T \Sigma K T=\Sigma K T$.

Proof. (a) $\Longrightarrow$ (b) (i) Suppose that $A \stackrel{\oplus}{\leq} B$ for some matrix $A$ such that $A^{\boxplus}$ exists. By Theorem 8 in [4], $A \leq B$. Since $B \neq O$, Theorem 1 asserts that there is an idempotent matrix $T \in \mathbb{C}^{r \times r}$ such that $A$ has the form (5). By Lemma 14 (a), $T \Sigma(T \Sigma)^{\dagger}=T$, so $T$ is hermitian and Lemma 12 leads

$$
A^{\boxplus}=U\left[\begin{array}{cc}
(T \Sigma K)^{\#} T \Sigma(T \Sigma)^{\dagger} & O \\
O & O
\end{array}\right] U^{*}=U\left[\begin{array}{cc}
(T \Sigma K)^{\#} T & O \\
O & O
\end{array}\right] U^{*} .
$$

Since $A \stackrel{\oplus}{\leq} B$, by definition we have $A^{\boxplus} A=A^{\boxplus} B$ and $A A^{\oplus}=B A^{\oplus}$. Using (18), (5), and (1) these last two equalities are equivalent to

$$
T \Sigma K(T \Sigma K)^{\#} T=\Sigma K(T \Sigma K)^{\#} T .
$$

Post-multiplying (19) by $\Sigma K$, the definition of the group inverse yields $T=$ $\Sigma K(T \Sigma K)^{\#} T$.

(b) (i) $\Longrightarrow$ (b) (ii) As we have shown in Remark 13, $T=\Sigma K(T \Sigma K)^{\#} T$ leads to $\Sigma K T=T \Sigma K T$. On the other hand, since $T$ is an idempotent hermitian

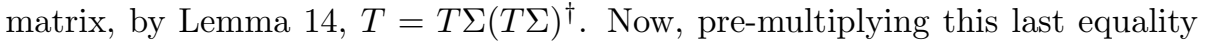
by $(T \Sigma)^{\dagger}$, we get $(T \Sigma)^{\dagger} T=(T \Sigma)^{\dagger}$.

(b) (ii) $\Longrightarrow$ (a) The existence of the core inverse of $A$ is guaranteed by Lemma 12. Taking into account that $T \Sigma K T=\Sigma K T$ and $(T \Sigma)^{\dagger} T=(T \Sigma)^{\dagger}$, from expressions (5) and (6) easy computations show that $A^{2}=B A$ and $A^{\dagger} A=A^{\dagger} B$. Hence, by equivalence (3.21) in [4] we arrive at $A \stackrel{\oplus}{\leq} B$.

The equivalence of (ii) and (iii) in (b) follows from Lemma 14. Note that this equivalence can be also deduced from the fact $A \stackrel{\oplus}{\leq} B \Longleftrightarrow A \leq * B, A \leq \# B$ (see [14, Proposition 5]) and Theorems 8 and 9.

Theorem 17 Let $A, B \in \mathbb{C}^{n \times n}$ be two matrices that have core inverses with $B \neq O$. Then the following statements are equivalent:

(a) $A \stackrel{\oplus}{\leq} B$.

(b) There is an idempotent hermitian matrix $Q \in \mathbb{C}^{n \times n}$ such that $A=Q B$ with $A Q=B Q$ and $r k(A)=r k(Q)$. 
Proof. (a) $\Longrightarrow$ (b) Suppose that $B$ is written as in (1). By Theorem 16, matrix $A$ can be expressed as $A=Q B$ with

$$
Q=U\left[\begin{array}{ll}
T & O \\
O & O
\end{array}\right] U^{*}
$$

where $T$ is an idempotent hermitian matrix such that $T \Sigma K T=\Sigma K T$. Then $Q^{2}=Q=Q^{*}$ and a simple computation allows us to verify that $Q B Q=B Q$. Moreover,

$$
r k(A)=r k\left(\left[\begin{array}{cc}
T \Sigma K & T \Sigma L \\
O & O
\end{array}\right]\right)=r k\left(\left[\begin{array}{cc}
T & O \\
O & O
\end{array}\right]\left[\begin{array}{cc}
\Sigma K & \Sigma L \\
O & I_{n-r}
\end{array}\right]\right) .
$$

Since $\Sigma K$ is nonsingular, $r k(A)=r k(T)=r k(Q)$.

(b) $\Longrightarrow$ (a) Suppose that there exists a unique idempotent hermitian matrix $Q$ such that $A=Q B, A Q=B Q$ and $r k(Q B)=r k(Q)$. Then $A^{2}=A Q B=$ $B Q B=B A$. It remains to show that $A=A A^{\dagger} B$ holds. Since $\mathcal{R}(A) \subseteq \mathcal{R}(Q)$ and $r k(A)=r k(Q)$, it follows that $\mathcal{R}(A)=\mathcal{R}(Q)$, so $Q B(Q B)^{\dagger}=Q Q^{\dagger}=Q$ because $Q$ is an orthogonal projector. Thus $A A^{\dagger} B=Q B(Q B)^{\dagger} B=Q B=A$ and hence $A^{\dagger} A=A^{\dagger} B$. Now, applying characterization (3.21) in [4] we arrive at $A \stackrel{\oplus}{\leq} B$.

Remark 18 The matrix $Q$ indicated in Theorem 17 is shown to be unique. Indeed, in order to show it, let us assume that

$$
Q_{1}=U\left[\begin{array}{cc}
X & Y \\
Z & S
\end{array}\right] U^{*}
$$

satisfies the same properties as $Q$. So, from

$$
\left[\begin{array}{cc}
T \Sigma K & T \Sigma L \\
O & O
\end{array}\right]=\left[\begin{array}{cc}
X & Y \\
Z & S
\end{array}\right]\left[\begin{array}{cc}
\Sigma K & \Sigma L \\
O & O
\end{array}\right]
$$

we have the following system

$$
\begin{aligned}
T \Sigma K & =X \Sigma K, \\
T \Sigma L & =X \Sigma L, \\
O & =Z \Sigma K, \\
O & =Z \Sigma L .
\end{aligned}
$$

By (20), $X=T$, and by (22), $Z=O$. Since $Q_{1}$ is hermitian, we obtain $Y=O$, and since $r k(A)=r k(T)=r k\left(Q_{1}\right)$, we get $S=O$. Hence, $Q_{1}=Q$.

The condition $A^{2} \stackrel{\oplus}{\leq} B^{2}$ has been studied in [4]. More information about the remaining powers is obtained in the next result.

Lemma 19 Let $A, B \in \mathbb{C}^{n \times n}$ matrices of indices at most 1 such that $A \stackrel{\oplus}{\leq} B$. Then the following conditions are equivalent. 
(a) $A B=B A$.

(b) $A^{2} \stackrel{\oplus}{\leq} B^{2}$.

(c) $A^{j} \stackrel{\oplus}{\leq} B^{j}$ for every integer $j \geq 2$.

Proof. (a) $\Longleftrightarrow$ (b) It was given in [4, Theorem 9].

(c) $\Longrightarrow$ (b) It is trivial.

(a) $\Longrightarrow$ (c) The $B=O$ case is trivial. Assume $B \neq O$ with $B$ written as in (1). By Theorem 16, there exists an idempotent hermitian matrix $T \in \mathbb{C}^{r \times r}$ such that $A$ has the form (5) and $T \Sigma K T=T \Sigma K$. Simple computations show that from $A B=B A$ we can deduce $T \Sigma K=\Sigma K T$. So, for every positive integer $j$, we have

$$
A^{j}=U\left[\begin{array}{cc}
T(\Sigma K)^{j} & T(\Sigma K)^{j-1} \Sigma L \\
O & O
\end{array}\right] U^{*} .
$$

Taking

$$
Q=U\left[\begin{array}{ll}
T & O \\
O & O
\end{array}\right] U^{*}
$$

we get $Q^{2}=Q=Q^{*}, A^{j}=Q B^{j}, A^{j} Q=B^{j} Q$, and $r k\left(A^{j}\right)=r k(Q)$. Now, Theorem 17 leads to $A^{j} \stackrel{\oplus}{\leq} B^{j}$.

When $A$ is an EP matrix the following result is obtained.

Proposition 20 Let $B \in \mathbb{C}^{n \times n}$ be a non-zero matrix written as in (1) that has core inverse. The following conditions are equivalent:

(a) There exists an EP matrix $A \in \mathbb{C}^{n \times n}$ such that $A \stackrel{\oplus}{\leq} B$.

(b) There is an idempotent hermitian matrix $T \in \mathbb{C}^{r \times r}$ such that

$$
A=U\left[\begin{array}{cc}
T \Sigma K & O \\
O & O
\end{array}\right] U^{*}
$$

with $\Sigma K T=T \Sigma K$.

Proof. (a) $\Longrightarrow$ (b) Applying Theorem 16 we have that there exists an idempotent hermitian matrix $T \in \mathbb{C}^{r \times r}$ such that $A$ has the form (5) with $T \Sigma K T=$ $\Sigma K T$. Since $A$ is $E P$, by Theorem 2 in [4] we have $A^{\boxplus}=A^{\#}$. Equating the expressions for $A^{\#}$ and $A^{\boxplus}$ given in Lemma 3 and Lemma 12 , respectively, we get

$$
\begin{aligned}
(T \Sigma K)^{\#} T \Sigma(T \Sigma)^{\dagger} & =(T \Sigma K)^{\#} \\
(T \Sigma K)^{\#} K^{-1} L & =O .
\end{aligned}
$$

It is clear that (25) reduces to $T \Sigma L=O$ and then $A$ has the required form. By Lemma 14, $T \Sigma(T \Sigma)^{\dagger}=T$. Then, from (24) we get $(T \Sigma K)^{\#} T=(T \Sigma K)^{\#}$. 
Pre-multiplying this last equation by $(T \Sigma K)^{2}$ we obtain $T \Sigma K T=T \Sigma K$. Since $T \Sigma K T=\Sigma K T$, the equality $\Sigma K T=T \Sigma K$ holds.

(b) $\Longrightarrow$ (a) It follows applying Theorem 16 and using the fact that $(T \Sigma K)^{\#}=$ $(\Sigma K)^{-1} T=(T \Sigma K)^{\dagger}$ (see Lemma 3).

In [14], it was proved that $A \stackrel{\oplus}{\leq} B \Longrightarrow A \overline{\leq} B$ and $A \leq^{s} B$ (where $A \leq^{s} B$ indicates the space pre-order given by $\mathcal{R}(A) \subseteq \mathcal{R}(B)$ and $\mathcal{R}\left(A^{*}\right) \subseteq \mathcal{R}\left(B^{*}\right)$ ). However, the converse is not valid as we can check, for example, by means of the following matrices

$$
A=\left[\begin{array}{ll}
1 & 1 \\
0 & 0
\end{array}\right], \quad B=I_{2}
$$

It is easy to see that $A \stackrel{\overline{\leq}}{\leq} B, A \leq^{s} B$ but $A \stackrel{\oplus}{\leq} B$ does not hold.

We have the following:

Theorem 21 Let $A, B \in \mathbb{C}^{n \times n}$ be matrices of indices at most 1. If $A \leq^{s} B$ and $A^{\oplus}=B^{\oplus}$ then $A \stackrel{\oplus}{\leq} B$.

Proof. It can be shown that $A \leq^{s} B$ is equivalent to $A=A B^{\boxplus} B=B B^{\boxplus} A$. So, $A A^{\boxplus}=A B^{\boxplus}$ implies $A A^{\boxplus} B=A B^{\boxplus} B=A$. Now pre-multiplying by $A^{\boxplus}$, we have $A^{\boxplus} B=A^{\boxplus} A$. Similarly, $A^{\boxplus} A=B^{\boxplus} A$ implies $B A^{\boxplus} A=B B^{\boxplus} A=A$. Now post-multiplying by $A^{\boxplus}$, we have $B A^{\boxplus}=A A^{\boxplus}$.

Example 22 Consider

$$
A=\left[\begin{array}{ll}
1 & 1 \\
0 & 0
\end{array}\right] \quad \text { and } \quad B=\left[\begin{array}{ll}
1 & 1 \\
0 & 1
\end{array}\right] .
$$

Then

$$
A^{\boxplus}=\left[\begin{array}{ll}
1 & 0 \\
0 & 0
\end{array}\right] \quad \text { and } \quad B^{\boxplus}=\left[\begin{array}{rr}
1 & -1 \\
0 & 1
\end{array}\right] .
$$

It can be seen that $A \stackrel{\oplus}{\leq} B$ but $A^{\boxplus} \neq B^{\oplus}$, even $A \leq s$ holds. Thus, the converse in Theorem 21 is not true.

The following example shows that if $A^{\oplus}=B^{\boxplus}$; it may not follow that $A \stackrel{\oplus}{\leq} B$.

Example 23 Consider

$$
A=\left[\begin{array}{ll}
1 & 1 \\
0 & 0
\end{array}\right] \quad \text { and } \quad B=\left[\begin{array}{ll}
1 & 0 \\
0 & 0
\end{array}\right] .
$$

It can be seen that

$$
A^{\boxplus}=\left[\begin{array}{ll}
1 & 0 \\
0 & 0
\end{array}\right]=B^{\boxplus}
$$

but the inequality $A \stackrel{\oplus}{\leq} B$ does not hold. Therefore, $A^{\boxplus}=B^{\boxplus}$ does not imply $A \stackrel{\oplus}{\leq} B$. 
When the converse in Theorem 15 is valid? Next result gives some information.

Theorem 24 Let $A, B \in \mathbb{C}^{n \times n}$ be matrices of indices at most 1 such that $A \stackrel{\triangleq}{\leq} B$. Then $A \stackrel{\oplus}{\leq} B$ if any of the following two items ( $a$ ) or (b) holds:

(a) $A$ is $E P$ and $B A^{\dagger} B=A$.

(b) $B^{*} A$ is hermitian and $A^{2}=B A$.

Proof. (a) By Theorem 2 in [2], $A \stackrel{\otimes}{\leq} B$ if and only if $A^{\dagger} \leq B^{\dagger}$. So, by Theorem 5.2.10 in [15], $A^{\dagger} \stackrel{*}{\leq} B^{\dagger}$. Now, Corollary 5.2.9 in [15] assures that $A \stackrel{*}{\leq} B$. Since $A$ is $E P, A \stackrel{\oplus}{\leq} B$ holds.

(b) Premultiplying $A A^{*} A=A B^{*} A$ by $A^{\dagger}$ and taking conjugate transpose we get $A^{*} A A^{\dagger} A=B^{*} A A^{\dagger} A$, that is $A^{*} A=B^{*} A=A^{*} B$, which yields $A * \leq B$. Now, $A^{2}=B A$ implies $A \stackrel{\oplus}{\leq} B$.

We close this section studying the reverse order law for the core generalized inverse. It was shown that this law does not hold in general [5]. Some previous results were given in [12] in the setting of EP matrices.

Theorem 25 Let $A, B \in \mathbb{C}^{n \times n}$ be matrices of indices at most 1 with $A \stackrel{\circledast}{\leq} B$. Suppose that $A, B$ and $T$ satisfy the conditions given in Theorem 16. Then the following equivalences hold.

(a) $(A B)^{\boxplus}=B^{\boxplus} A^{\boxplus}$ if and only if $\left(T(\Sigma K)^{2}\right)^{\#} T=(\Sigma K)^{-2} T$.

(b) $(B A)^{\boxplus}=A^{\boxplus} B^{\boxplus}$ if and only if $T=\Sigma K(\Sigma K T \Sigma K)^{\#} \Sigma K T \Sigma(\Sigma K T \Sigma)^{\dagger} \Sigma K$.

Proof. By Theorem 16, there exists an idempotent hermitian matrix $T \in \mathbb{C}^{r \times r}$ such that $A$ has the form (5) with $T=\Sigma K(T \Sigma K)^{\#} T$. By Lemma 12, Lemma 14 and Lemma 2 in [4] we have

$$
A^{\boxplus}=U\left[\begin{array}{cc}
(T \Sigma K)^{\#} T & O \\
O & O
\end{array}\right] U^{*} \quad \text { and } \quad B^{\boxplus}=U\left[\begin{array}{cc}
(\Sigma K)^{-1} & O \\
O & O
\end{array}\right] U^{*} \text {. }
$$

Now, condition $T=\Sigma K(T \Sigma K)^{\#} T$ yields

$$
B^{\boxplus} A^{\boxplus}=U\left[\begin{array}{cc}
(\Sigma K)^{-1}(T \Sigma K)^{\#} T & O \\
O & O
\end{array}\right] U^{*}=U\left[\begin{array}{cc}
(\Sigma K)^{-2} T & O \\
O & O
\end{array}\right] U^{*} .
$$

On the other hand, applying again Lemma 12 to

$$
A B=U\left[\begin{array}{cc}
(T \Sigma K)(\Sigma K) & (T \Sigma K)(\Sigma L) \\
O & O
\end{array}\right] U^{*}
$$

and using that Lemma 14 leads to $T \Sigma K \Sigma(T \Sigma K \Sigma)^{\dagger}=T$, we have

$$
(A B)^{\boxplus}=U\left[\begin{array}{cc}
\left(T(\Sigma K)^{2}\right)^{\#} T & O \\
O & O
\end{array}\right] U^{*} .
$$


Now, the equivalence in (a) follows immediately from (26) and (27). Similar computations yield (b).

Corollary 26 Let $A, B \in \mathbb{C}^{n \times n}$ be matrices of indices at most 1 . If $A \stackrel{\oplus}{\leq} B$ and $A B^{2}=B^{2} A$ then $(A B)^{\boxplus}=B^{\boxplus} A^{\boxplus}$.

Proof. Following the notation used in Theorem 25, we can observe that

$$
A B^{2}=U\left[\begin{array}{cc}
T(\Sigma K)^{3} & T(\Sigma K)^{2} \Sigma L \\
O & O
\end{array}\right] U^{*}
$$

and

$$
B^{2} A=U\left[\begin{array}{cc}
(\Sigma K)^{2} T \Sigma K & (\Sigma K)^{2} T \Sigma L \\
O & O
\end{array}\right] U^{*} .
$$

Since $\Sigma K$ is nonsingular, $A B^{2}=B^{2} A$ if and only if $T(\Sigma K)^{2}=(\Sigma K)^{2} T$. Then, it can be proved that $\left(T(\Sigma K)^{2}\right)^{\#}=(\Sigma K)^{-2} T$ holds and so $(A B)^{\boxplus}=B^{\boxplus} A^{\boxplus}$.

Notice that the converse in Corollary 26 is false. Matrices $A$ and $B$ exhibid in Examples 22 and 23 provide two counterexamples. While in the first case, condition $A \stackrel{\oplus}{\leq} B$ is fulfilled, in the second one it does not hold.

Notice that, in particular, if $A \stackrel{\oplus}{\leq} B$ and $A B=B A$ then $A^{\boxplus} B^{\boxplus}=B^{\boxplus} A^{\boxplus}$.

\section{$5 \quad$ When is the property $B-A \stackrel{\oplus}{\leq} B$ valid?}

A property that was studied under minus, star, and sharp partial orders is the following: $A$ is below $B$ under one of these partial orders if and only if $B-A$ is below $B$ under the same order. In [4], the authors showed that $A \stackrel{\oplus}{\leq} B \nRightarrow$ $B-A \stackrel{\oplus}{\leq} B$ for the core partial order. It is also easy to see that $B-A \stackrel{\oplus}{\leq} B$ does not imply $A \stackrel{\oplus}{\leq} B$ as it can be checked if we take, for example,

$$
A=\left[\begin{array}{ll}
0 & 0 \\
0 & 1
\end{array}\right] \quad \text { and } \quad B=\left[\begin{array}{ll}
1 & 1 \\
0 & 1
\end{array}\right] .
$$

We can still give some more information.

Proposition 27 Let $A, B \in \mathbb{C}^{n \times n}$ be two matrices such that $A, B$, and $B-A$ have core inverses and let $B$ be a non-zero matrix written as in (1) that satisfies $A \stackrel{\oplus}{\leq} B$ where

$$
A=U\left[\begin{array}{cc}
T_{A} \Sigma K & T_{A} \Sigma L \\
O & O
\end{array}\right] U^{*}
$$

and the idempotent hermitian matrix $T_{A}$ satisfies $T_{A} \Sigma K T_{A}=\Sigma K T_{A}$ as Theorem 16 asserts. Then the following statements are equivalent. 
(a) $B-A \stackrel{\oplus}{\leq} B$.

(b) $T_{A} \Sigma K=\Sigma K T_{A}$.

Proof. Under the assumptions and the above notations it is clear that

$$
B-A=U\left[\begin{array}{cc}
\left(I-T_{A}\right) \Sigma K & \left(I-T_{A}\right) \Sigma L \\
O & O
\end{array}\right] U^{*} .
$$

On the other hand, by Theorem 16 , the condition $B-A \stackrel{\oplus}{\leq} B$ is equivalent to the existence of an idempotent hermitian matrix $T_{B-A} \in \mathbb{C}^{r \times r}$ such that

$$
B-A=U\left[\begin{array}{cc}
T_{B-A} \Sigma K & T_{B-A} \Sigma L \\
O & O
\end{array}\right] U^{*}
$$

and $T_{B-A} \Sigma K T_{B-A}=\Sigma K T_{B-A}$. Comparing expressions (28) and (29) we obtain $T_{B-A}=I-T_{A}$. So, $\left(I-T_{A}\right) \Sigma K\left(I-T_{A}\right)=\Sigma K\left(I-T_{A}\right)$, which is equivalent to $T_{A} \Sigma K T_{A}=T_{A} \Sigma K$. Since $T_{A} \Sigma K T_{A}=\Sigma K T_{A}$, we have shown that $B-A \stackrel{\oplus}{\leq} B$ is equivalent to $T_{A} \Sigma K=\Sigma K T_{A}$.

Taking into account that $A=B-(B-A)$ we can immediately state the following result.

Proposition 28 Let $A, B \in \mathbb{C}^{n \times n}$ be two matrices such that $A, B$ and $B-A$ have core inverses and let $B$ be a non-zero matrix written as in (1) that satisfies $B-A \stackrel{\oplus}{\leq} B$ where

$$
B-A=U\left[\begin{array}{cc}
T_{B-A} \Sigma K & T_{B-A^{\Sigma} L} \\
O & O
\end{array}\right] U^{*}
$$

and the idempotent hermitian matrix $T_{B-A}$ satisfies $T_{B-A} \Sigma K T_{B-A}=\Sigma K T_{B-A}$ as Theorem 16 asserts. Then the following statements are equivalent.

(a) $A \stackrel{\oplus}{\leq} B$.

(b) $T_{B-A} \Sigma K=\Sigma K T_{B-A}$.

Let $A, B \in \mathbb{C}^{n \times n}$ be two matrices that have core inverse such that $A \stackrel{\oplus}{\leq} B$. Without loss of generality, we can assume that $A$ is written as

$$
A=U_{A}\left[\begin{array}{cc}
\Sigma_{A} K_{A} & \Sigma_{A} L_{A} \\
O & O
\end{array}\right] U_{A}^{*}
$$

where $U_{A}, \Sigma_{A}, K_{A}$, and $L_{A}$ satisfy the conditions in the Hartwig-Spindelböck decomposition. Then, applying Lemma 3 in [4] we have

$$
B=U_{A}\left[\begin{array}{cc}
\Sigma_{A} K_{A} & \Sigma_{A} L_{A} \\
O & Z
\end{array}\right] U_{A}^{*},
$$

for some matrix $Z$ which has a core inverse. Notice that this matrix $Z$ is unique. Under this notation we can state the following result. 
Lemma 29 Let $A, B \in \mathbb{C}^{n \times n}$ be two matrices that have core inverse such that $A \stackrel{\oplus}{\leq} B$ written as in (30) and (31), respectively. Then $B-A \stackrel{\oplus}{\leq} B$ if and only if $L_{A} Z=O$.

Proof. Under the above notation, we get

$$
B-A=U_{A}\left[\begin{array}{ll}
O & O \\
O & Z
\end{array}\right] U_{A}^{*}
$$

Since $(B-A)^{\oplus}=(B-A)^{\#}(B-A)(B-A)^{\dagger}$, we have

$$
(B-A)^{\boxplus}=U_{A}\left[\begin{array}{cc}
O & O \\
O & Z^{\#} Z Z^{\dagger}
\end{array}\right] U_{A}^{*}=U_{A}\left[\begin{array}{cc}
O & O \\
O & Z^{\oplus}
\end{array}\right] U_{A}^{*} .
$$

Now, it is easy to see that the equalities $(B-A)^{\oplus}(B-A)=(B-A)^{\oplus} B$, $(B-A)(B-A)^{\oplus}=B(B-A)^{\oplus}$ are equivalent to $L_{A} Z^{\boxplus}=O$, that is $L_{A} Z^{\#}=O$.

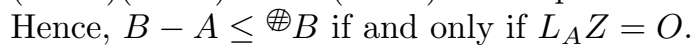

We remark that if $A$ is $E P$ (that is $\left.L_{A}=O\right)$ then $A \stackrel{\oplus}{\leq} B$ implies $B-A \stackrel{\oplus}{\leq} B$.

Lemma 30 Let $A, B \in \mathbb{C}^{n \times n}$ be such that $A$ and $B-A$ are EP matrices. Then $A \stackrel{\oplus}{\leq} B$ if and only if $B-A \stackrel{\oplus}{\leq} B$.

Proof. By Theorem 7 in [4], $A \stackrel{\oplus}{\leq} B$ if and only if $A \stackrel{*}{\leq} B$ since $A$ is $E P$. Now, Theorem 5.2.3 in [15] asserts the equivalence of $A \stackrel{*}{\leq} B$ and $B-A \stackrel{*}{\leq} B$. Again, taking into account that $B-A$ is $E P, B-A \stackrel{*}{\leq} B$ is equivalent to $B-A \stackrel{\oplus}{\leq} B$.

In [4], it is seen that neither $A \stackrel{\oplus}{\leq} B$ implies $A \stackrel{\#}{\leq} B$ nor $A \stackrel{\#}{\leq} B$ implies $A \stackrel{\oplus}{\leq} B$ are valid in general. Despite this, from Theorem 4, Theorem 5, Theorem 16, Proposition 27 and Proposition 28, the following result is true.

Lemma 31 Let $A, B \in \mathbb{C}^{n \times n}$ be matrices such that $A$ and $B-A$ have indices at most 1 . The following statements hold.

(a) $A \stackrel{\oplus}{\leq} B$ and $A B=B A$ if and only if $A \stackrel{\#}{\leq} B$ and $A^{*} A=A^{*} B$.

(b) $A \stackrel{\oplus}{\leq} B$ and $A B^{*}$ hermitian if and only if $A \stackrel{*}{\leq} B$ and $A^{2}=B A$.

(c) If $A \stackrel{\oplus}{\leq} B$ then $B-A \stackrel{\oplus}{\leq} B$ is equivalent to $A \stackrel{\#}{\leq} B$.

(d) If $B-A \stackrel{\oplus}{\leq} B$ then $A \stackrel{\oplus}{\leq} B$ is equivalent to $B-A \stackrel{\#}{\leq} B$.

Even more, for $E P$ matrices we can state the following result.

Corollary 32 Let $A, B \in \mathbb{C}^{n \times n}$ be two matrices such that $A$ and $B-A$ are $E P$. Then the following statements are equivalent. 
(a) $A \stackrel{\oplus}{\leq} B$.

(b) $A \stackrel{*}{\leq} B$.

(c) $A \stackrel{\#}{\leq} B$.

(d) $B-A \stackrel{\oplus}{\leq} B$.

(e) $B-A \stackrel{*}{\leq} B$.

(f) $B-A \stackrel{\#}{\leq} B$.

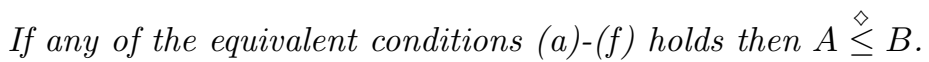

Proof. It is a direct consequence of Lemma 30 and Theorem 7 in [4].

\section{References}

[1] J.K. Baksakary, O.M. Baksalary, X. Liu, Further properties of the star, left-star, right-star and minus partial orderings, Linear Algebra and its Applications, 375, 83-94, 2003.

[2] J.K. Baksalary, J. Hauke, A further algebraic version of Cochran's theorem and matrix partial orderings, Linear Algebra and its Applications, 127, 157-169, 1990.

[3] O.M. Baksalary, G.P.H. Styan, G. Trenkler, On a matrix decomposition of Hartwig and Spindelböck. Linear Algebra and its Applications, 430, 2798$2812,2009$.

[4] O.M. Baksalary, G. Trenkler, Core inverse of matrices, Linear and Multilinear Algebra, 58, 681-697, 2010.

[5] O.M. Baksalary, G. Trenkler, Reverse Order Law for the Core Inverse: Problem 48-1, IMAGE, 49, 46-47, 2012.

[6] A. Ben-Israel, T. Greville, Generalized Inverses: Theory and Applications, John Wiley \& Sons, Second Edition, 2003.

[7] S.L. Campbell, C.D. Meyer Jr., Generalized Inverse of Linear Transformations. Dover, New York, Second Edition, 1991.

[8] J. Groß, J. Hauke, A. Markiewicz, Partial orderings, preorderings, and the polar decomposition of matrices, Linear Algebra and its Applications, 289, 161-168, 1999.

[9] R.E. Hartwig, How to partially order regular elements, Math. Japon., 25, $1-13,1980$. 
[10] R.E. Hartwig, K. Spindleböck, Matrices for which $A^{*}$ and $A^{\dagger}$ commmute, Linear and Multilinear Algebra, 14, 241-256, 1984.

[11] L. Lebtahi, P. Patrício, N. Thome, The diamond partial order in rings, Linear and Multilinear Algebra, DOI: http://dx.doi.org/10.1080/03081087.2013.779272, 2013.

[12] S.B. Malik, Some more properties of core partial order, submitted.

[13] C.D. Meyer, Matrix Analysis and Applied Linear Algebra. SIAM, Philadelphia, 2000.

[14] J. Mielniczuk, Note on the core matrix partial ordering, Discussiones Mathematicae, Probability and Statistics, 31, 71-75, 2011.

[15] S.K. Mitra, P. Bhimasankaram, S.B. Malik, Matrix partial orders, shorted operators and applications. World Scientific Publishing Company, 2010.

[16] D. Mošić, D.S. Djordjević, Reverse order law for the group inverse in rings, Applied Mathematics and Computation, 219, 5, 2526-2534, 2012.

[17] P. Patricio, A. Veloso da Costa, On the Drazin index of regular elements, Central European Journal of Mathematics, 7, 2 200-205, 2009.

[18] D. S. Rakić, D. S. Djordjević, Space pre-order and minus partial order for operators on Banach spaces, Aequationes Mathematicae DOI: 10.1007/s00010-012-0133-2, 2012.

[19] M. Tošić, D.S. Cvetković-Ilić, Invertibility of a linear combination of two matrices and partial orderings, Applied Mathematics and Computations, 218, 4651-4657, 2012. 